\title{
Ordering Effects and Percolation in the Structure Formation Process of the Oriented Polyolefin Porous Films
}

\author{
Galina Kazimirovna Elyashevich, ${ }^{3,{ }^{*}}$ Dmitrii Vladimirovich Novikov, ${ }^{3}$ \\ Ivan Sergeevich Kuryndin, ${ }^{3}$ Andreja Jelen ${ }^{2}$ and Vili Bukošek ${ }^{1, *}$ \\ ${ }^{1}$ Faculty for Natural Sciences and Engineering, University of Ljubljana,Snežniška c. 5, Ljubljana, 1000 Slovenia \\ ${ }^{2}$ Jožef Stefan Institute, Jamova cesta 39, Ljubljana, 1000 Slovenia \\ ${ }^{3}$ Institute of Macromolecular Compounds, Russian Academy of Sciences,Bolshoi pr. 31, St. Petersburg, 199004 Russia \\ *Corresponding author:E-mail:elya@hq.macro.ru; vili.bukosek@ntf.uni-lj.si
}

Received: 12-07-2017

\begin{abstract}
Structure transitions and mechanism of the formation of superlattices lamellae in microporous polyolefin (polyethylene and polypropylene) films obtained in the process based on polymer melt extrusion followed by annealing, uniaxial extension and thermal fixation have been studied by statistical analysis of electron microscopy images of the film surface. The structure of the porous films prepared in the multistage process has been studied by SEM, gravimetry and permeability measurements. It has been shown that the pore formation at the stage of uniaxial extension is accompanied by the ordering of lamellae and their self-organization controlled by spin draw ratio and annealing temperature. It was established that an increase of these parameters lead to the transition of disorder-order type. The effect of preparation conditions on the ordering process of regular spatial lattices of lamellae has been discussed.
\end{abstract}

Keywords: Percolation, self-organization, through permeability, porous films, polyethylene, polypropylene

\section{Introduction}

At present time a large number of porous systems containing microscopic pores have been prepared and studied; and among them, polyolefin films are the most promising materials. Owing to high chemical resistance to various media, they are widely used as gas and liquid permeable membrane materials in medical, chemical, and food industries. Microporous polymer films have a number of advantages over porous systems based on inorganic substances, namely: easy manufacturing, small thickness and hence lower resistance to mass transfer, and high elasticity.

Flexible-chain polyolefins such as high density polyethylene $^{1,2}$ and polypropylene ${ }^{3-6}$ are capable to form the oriented lamellar structures during crystallization of polymer melts under extension. The thermal treatment of these samples at temperatures close to the melting temperature of a polymer leads to an increase in the degree of crystallinity and a decrease in the number of tie chains in amorphous regions between lamellae, and, finally, almost all tie chains become stressed. In result of such structure formation the materials (films and fibers) acquire so-called "hard elastic" mechanical properties, that is, high elastic modulus and ability to large reversible deformations. ${ }^{4,7}$ Due to a very low number of tie chains in amorphous regions the pores appear between lamellae at uniaxial extension of hard elastic samples in air at room temperature. Porous films with well developed networks of through flow channels may be used as microfiltration membranes, separators in galvanic cells, and elastic substrates in composite systems. ${ }^{8-13}$

Up to now, there have been few published papers ${ }^{5,14}$ concerning the analysis of the porousfilm preparation process, at which transition from individual pores to a connected network of through flow channels is observed. In ${ }^{14}$ it was shown that the formation of through pores in the samples prepared in the process based on melt extrusion occurs via the percolation mechanism..$^{15}$ The task of the work was the investigation of the effect of pore formation process conditions on the percolation transitions and or- 
dering effects at the self-organization of lamellar structure in the polyolefin porous films.

\section{Experimental}

The process used to prepare the microporous polyolefin films consists of four stages: (1) melt extrusion, (2) annealing of extruded films, (3) uniaxial extension of the annealed films (pore formation stage), and (4) thermal fixation of the porous structure. ${ }^{2,5,14}$ The films are characterized by a multilevel (multifractal) structure. ${ }^{5}$ Such structure is formed by stacks of crystalline lamellae arranged normally to the orientation (extension) direction of samples, so that molecular chains in crystallites are oriented in the extension direction. Lamellae stacks are connected by thin "bridges" (stressed ties) and form the framework of the solid-phase percolation cluster. ${ }^{16}$ In the process of uniaxial extension, discontinuities (pores) appear in such filmsas a result of moving apart and bending of lamellae between the bridges of tie chains. It was shown that the number and size of pores grow with an increase in the degree of extension that leads to the coalescence of pores and the formation of through flow channels, i.e., the films become filtration membranes permeable to liquids. It was also found that the overall porosity $(P)$ and the permeability $(G)$ of the membranes can be controlled by varying the spin draw ratio $(\lambda)$ at the polymer melt extrusion, the annealing temperature $\left(T_{\text {ann }}\right)$ of extruded films and degree of uniaxial extension $(\varepsilon)$ at the pore formation stage. ${ }^{17}$

Two structure self-ordering types are implemented in the prepared microporous films. The first type includes the cooperative percolation over pores (the formation of through channels) or the geometrical phase transition occurring upon reaching a critical degree of the overall porosity. The second type is associated with the periodic spatial superlattice of lamellae due to the disorder-order transition and ordering of particle aggregates (stacks of lamellae).The percolation transition leads to the formation of a liquid-permeable porous structure and the self-organization of lamellae at increasing the control parameters of the process: $\lambda, T_{\text {ann }}$ and $\varepsilon$.

Commercial grades of linear PE $\left(M_{w}=170000, M_{w}\right.$ ' $\left.M_{n}=4-5\right)$ and isotactic PP $\left(M_{w}=380000, M_{w} / M_{n}=4-5\right)$ was used for porous films preparation. The melt was crystallized in air. The degree of melt orientation was varied by the spin draw ratio $\lambda$. Extruded films were annealed under isometric conditions, i.e. at fixed ends of the film, to prevent their shrinkage at heating. The porous structure of membranes was formed at uniaxial extension of annealed films at room temperature. At the final stage, thermal fixation of the porous samples was performed for relaxation of inner stresses resulting from extension.

The permeability of porous films $G$ was determined by measuring the liquid (ethanol) flow rate through the film. ${ }^{8}$
An important characteristic of porous systems is overall porosity, i.e., the fraction of the sample volume occupied by pores. In the microporous films under investigation all type of pores, namely, the open-cell, closed and through pores, contribute to the overall porosity $P$ which was determined by the gravimetric method.

$$
P=\left[\left(\rho-\rho_{\mathrm{p})} / \rho\right] \times 100 \%,\right.
$$

Where $\rho$ is the density of a dense (nonporous) film, being equal to 950 and $900 \mathrm{~kg} / \mathrm{m}^{3}$ for PE and PP films, respectively; and $\rho_{\mathrm{p}}$ is the density of a porous film determined by weighing.

The images of the surface of membrane samples were obtained on a FE-SEM SUPRA 35 scanning electron microscope (Ziess, Germany).

Statistic analysis of SEM images was carried out using the cluster two-phase model on a square lattice with the ratio $r / \xi \approx 0.1$, where $r$ is the distance between sites and $\xi$ is the correlation length of phase clusters. Within this model, we calculated the average lattice density $\Omega_{\mathrm{p}}$ of porous phase clusters (porous phase fraction in two-dimensional space) and the radial distribution functions $g(R)$ of the cluster lattice density. The effective size $d$ and fractal dimension $D$ of porous phase clusters were determined by the initial region $(R<d)$ of the fall of curves $g(R)$ using the power-law asymptotic, $g(R) \sim R^{D-2}$.

The correlation length $\xi$ was identified with the abscissa of the point at the maximum of the direction averaged radial density distribution function $g(R)$ of the porous phase clusters. When the point of the maximum was absent, the correlation length was identified with the point at which the function $g(R)$ reached the asymptote $g(R)=1$.

The angular dependences (indicatrices) of the lattice fractal density $\rho s$ of the solid phase clusters were obtained according to the procedure described on the scale of a rectangle with area $2 r \xi$ centered at the occupied lattice site.

The degree of ordering of the lamellae in the films $\varphi$, was quantitatively estimated using the coordination order parameter along the extension axis of the films:

$$
\varphi=\left|L_{\mathrm{s} \|}-L_{\mathrm{p} \|}\right| /\left(L_{\mathrm{s}||} L_{\mathrm{p} \|}\right)^{1 / 2},
$$

where $L_{\mathrm{s} \|}$ and $L_{\mathrm{p} \|}$ are the periods of alternation of the lattice densities of the solid phase and the porous phase clusters, respectively, along the $s$ axis.

The regular spatial lattice of particles corresponds to the parameter $\varphi=0$; with an increase in the coordination disorder, the parameter $\varphi$ increases. The periods $L_{s \|}$ and $L_{\mathrm{p} \|}$ were determined using the functions $g(R)_{\|}$calculated in the direction of this axis. The thickness $l_{\|}$of solid phase particles was calculated as $l_{\|}=0.8 R_{\min }$, where $R_{\min }$ is the position of the first minimum of the corresponding function $g(R)_{\|}$. 


\section{Results and Discussion}

The microporous films prepared by the method used in this study are characterized by a relief-like strongly developed surface. This pattern arises at uniaxial extension stage in result of stress release due to the through pores appearance. As can be seen from SEM images, the surface relief of the PE and PP films (Figure 1)is designed by two types of structural elements; namely, extended large comblike structures arranged perpendicular to the direction of orientation of the film and thin "bridges" aligned parallel to the film orientation direction and connecting the comblike structures.
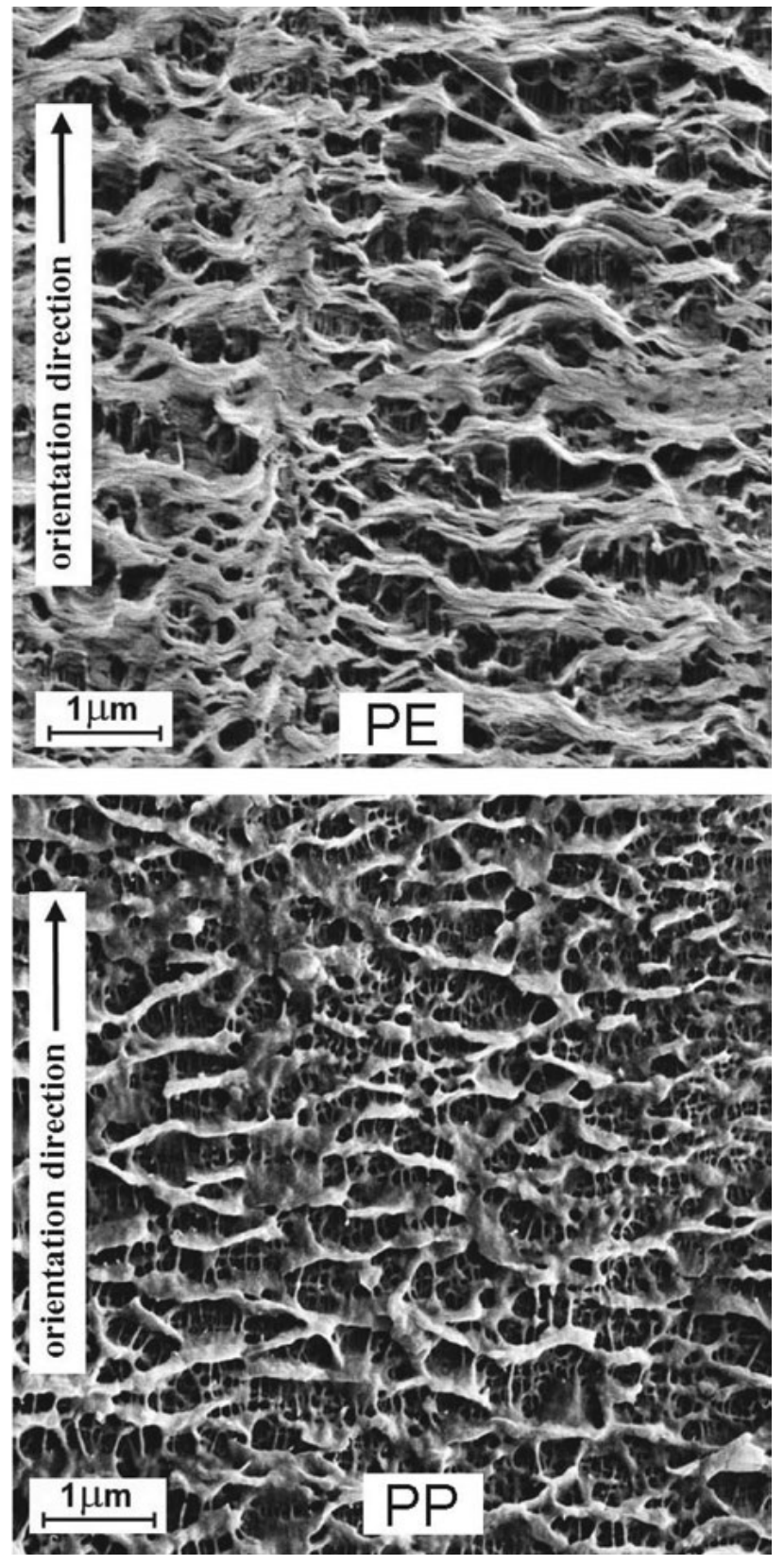

Figure 1. SEM images of the PE and PP porous films surface
Deeper regions (pores) are seen between these structural formations on the sample surface. The PP samples are characterized by smaller sizes of the structural elements of the surface relief and by their considerably larger number as compared to the PE films. These characteristics of the surface structure indicate that the PP films have a more developed surface relief than the PE ones. This is confirmed by the measurements of specific surface, which is equal to 41 and $83 \mathrm{~m}^{2} / \mathrm{g}$ for PE and PP microporous films, respectively.

\section{1. Effect of Spin Draw Ratio on the Ordering Transitions}

Due to the orientation efforts at the melt extrusion the periodic superlattice of oriented lamellae is formed in the films via the universal mechanism of particle self-ordering, controlled by the spin draw ratio $\lambda$. At the stages of extrusion and annealing which is performed at isometric conditions, lamellae are organized in an ensemble of oriented supramolecular structures. Under uniaxial extension of the films prepared at these stages this ensemble transforms to a spatial network of smaller particles, i.e., lamellae stacks (superlattices of lamellae), due to irreversible splitting of structons. The superlattice formation can be considered as the disorder-order transition, which leads to ordering of the multilevel supramolecular polymer structure. At increasing of $\lambda$ the size and degree of the aggregates ordering increase. The transformation of the microporous films' surface texture with variations in spin draw ratio is caused by the change in the relative contribution from the stacks of lamellae and stressed ties connecting them to the spatial distribution of the density of the solid phase cluster.

A typical property of the microporous polyolefin films under study is the percolation effects which are associated with the formation of a fractal structure of the surface. The fractal cluster of the solid phase is arisen near the threshold of percolation in result of the coalescence of pores and appearance of through flow channels. Percolation mechanism of the through pores formation is demonstrated by the dependence of the permeability $G$ (flow rate through the membrane) on $\lambda$ which exhibits a percolation threshold of $\lambda$ for permeability at threshold value of the overall porosity $P$ (the sample volume fraction occupied with pores) (Figure 2).

For PE films the threshold is reached at $P^{\star}=(30 \pm 2)$ $\%$, for PP ones $P^{\star}=(23 \pm 2) \%$. The difference between the values of $P^{\star}$ for $\mathrm{PE}$ and $\mathrm{PP}$ is connected with the structure details of PE films. This result is in accordance with percolation theory ${ }^{15}$ which gives for percolation threshold $2530 \%$.

The transition through the percolation threshold at $P$ $>P^{\star}$ is accompanied bythe formation of a percolation cluster of the porous phase in the material. On the film surface, this transition corresponds to an increase in the correla-

Elyashevich et al.: Ordering Effects and Percolation ... 

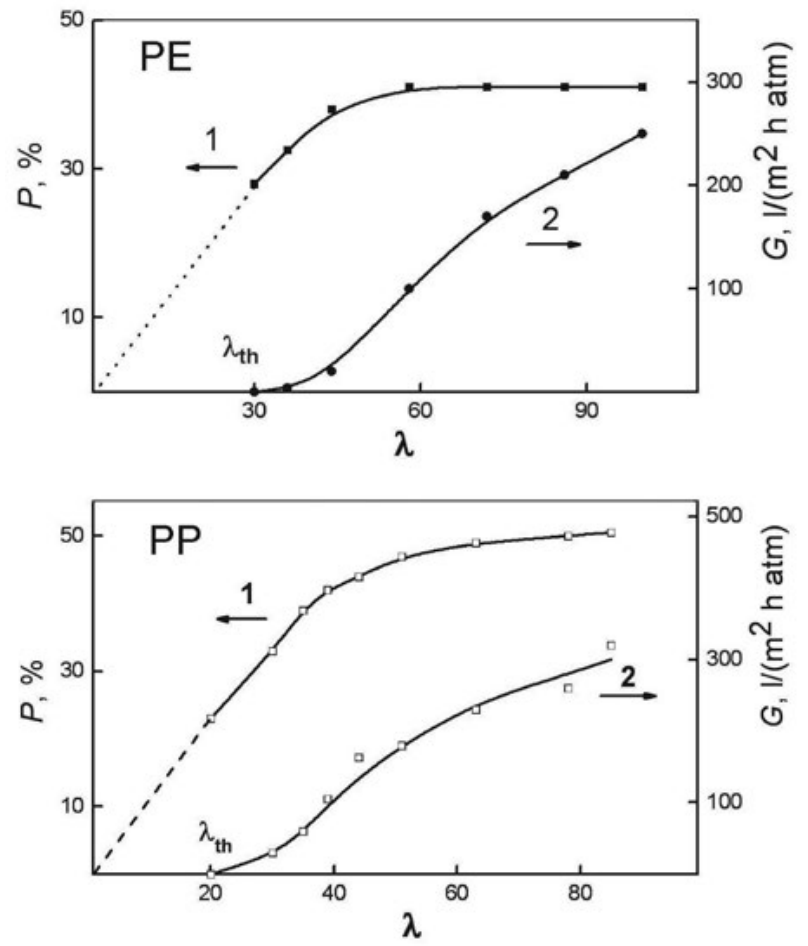

Figure 2. Plots of overall porosity P (1) and permeability G (2) of porous $\mathrm{PE}$ and $\mathrm{PP}$ films vs. spin draw ratio $\lambda$.

tion length $\xi$, a decrease in the total anisotropy of the density distribution $\rho s$ of the solid phase clusters, and a disordering of the average density distribution of the porous phase clusters. The above phenomena have a common character and are observed in the computer implementation of the lattice clusters models.

The orientational order of lamellae and the degree of orientation of polymer chains increase with increasing $\lambda$. This growth is nonlinear, which is indicated by both the nonmonotonic dependence of the correlation length on $\lambda$ for investigated samples and a significant scatter of the data on birefringence in PE porous films, obtained by changing the parameter $\lambda .{ }^{18}$ It can be assumed that the nonlinear in $\lambda$ orientation behavior reflects different conditions of their structure formation depending on parameters of melt extrusion process. In this case, the transition from one mode to another is accompanied by a change in the orientation of molecules and supramolecular structures themselves and also porous phase clusters. Of particular interest is the disorder-order orientation transition on the $\lambda$ scale, associated with the formation of oriented through channels.

Microporous PE films were prepared at variation of $\lambda$ in the range 24-69. The structural parameters and porous structure transformation have been investigated by statistical treatment of the SEM images. It was obtained that the lattice density fraction $\Omega_{\mathrm{s}}$ of the solid phase on the surface of microporous PE films corresponds in two-dimensional mapping to the infinite cluster of the particles at $\Omega_{\mathrm{s}} \geq \Omega^{*}$, where $\Omega^{*}$ is the critical value at the percolation threshold $(0.45 \pm 0.03) .{ }^{19}$ In its turn, the porous structure of the films is transformed upon varying parameter $\lambda$, and the transition from discrete pores $\left(\Omega_{\mathrm{p}}<\Omega^{*}\right)$ to through channels $\left(\Omega_{\mathrm{p}}\right.$ $>\Omega^{*}$ ) occurs at $\lambda>29$. The existence of the critical value of $\lambda$, which provides percolation in PE membranes, was previously found in. ${ }^{14}$

Radial functions $g(R)$ of the clusters density distribution of the phases in the general case is typical for the structurally nonuniform systems and nonmonotonically approach the straight line $g(R)=1$ (Figure 3a) as $R$ increases. This straight line is reached at $R=\xi$, where $\xi$ is the correlation radius of density fluctuations. ${ }^{19}$ It should be expected that the values of $\xi$ for two mutually complementary percolation clusters coincide (Figures $3 b, 3 c$ ).
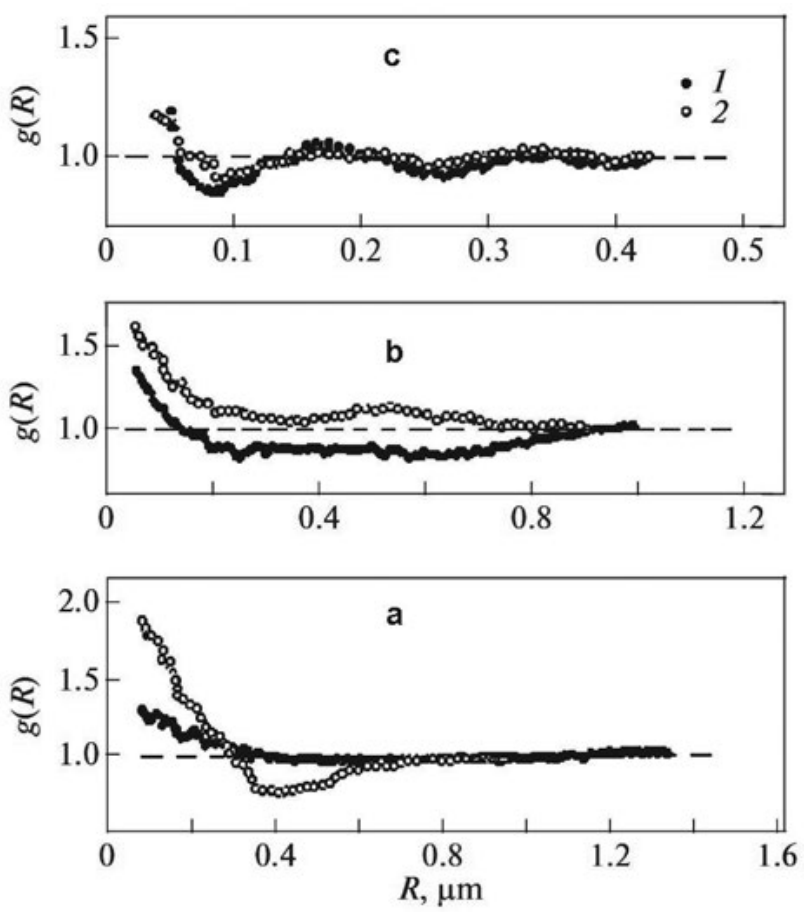

Figure 3. Distribution functions $\mathrm{g}(\mathrm{R})$ of the clusters density along the directions: solid phase (1) and porous phase (2) for $\lambda=24$ (a), 36 (b) and 69 (c).

At the same time as lattice density $\Omega_{\mathrm{p}}$ of the cluster of the particles decreases, the value of $\xi$ increases (Table 1 ).

For the PE film obtained at $\lambda=69$ the periodic oscillation of the functions $g(R)$ near the value $g(R)=1$, and

Table 1. The structural parameters of the porous PE films.

\begin{tabular}{ccccc}
\hline \multicolumn{1}{c}{ Solid phase } & \multicolumn{2}{c}{ Pores } \\
& $\boldsymbol{\xi}, \boldsymbol{\mu \mathrm { m }}$ & $\boldsymbol{d}, \boldsymbol{\mu m}$ & $\boldsymbol{\Omega}_{\mathbf{p}}$ & $\boldsymbol{d}, \boldsymbol{\mu m}$ \\
\hline 24 & 0.71 & 0.6 & 0.36 & 0.35 \\
29 & 0.25 & 0.13 & 0.30 & 0.18 \\
36 & 0.54 & 0.28 & 0.46 & 0.38 \\
42 & 0.58 & 0.80 & 0.42 & 0.62 \\
69 & - & 0.09 & 0.57 & 0.09 \\
\hline
\end{tabular}


functions $g(R)$ almost coincide both for the solid phase and for pores which indicates the formation of the uniform periodic structure of microporous PE films. The infinite cluster is transformed into the two-dimensional quasilattice of oriented particles, and the superlattice is constructed of ordered stacks of lamellae, so that each stack involves $~ 2.5$ lamellae. The mean sizes of the particles (stacks of lamellae) and pores in the direction perpendicular to the orientation axis coincide.

The formation of through channels at increasing of $\lambda$ is associated with ordering the particles in the orientation direction of the samples. Functions $g(R)$ of clusters of the phases along the orientation direction indicate that the periodicity of alternation of the particles with period $L_{\|}$appears at the scale of the correlation radius $\xi$. It was calculated that the value of $L_{\|}$substantially exceeds both the thickness of a single lamella $(30 \mathrm{~nm})$ and the long pe$\operatorname{riod}(35 \mathrm{~nm}){ }^{20}$ Thus, the anisometric particles that are revealed by SEM on the film surface are the fragments of the solidified polymer material involving the stacks of lamellae. The pattern of the surface is similar to the internal lamellar structure of porous samples; however, they are characterized by a larger scale of structural elements. ${ }^{20,21}$ Periods $L_{\|}$of alternation of the particles and pores in the orientation direction for the porous sample prepared at $\lambda=69$ coincide and correspond to the average period $L=170 \mathrm{~nm}$.

Structure transformations in PP porous films were investigated for the samples prepared at $\lambda=44$ and 78. In the samples, prepared at $\lambda=78$, the $3 \mathrm{D}$ regular lattice of lamellae stacks and also the system of oriented through flow channels providing the highest permeability to the sample are formed. In 2-dimensional images the regular scale lattice of lamellae is characterized by a coincidence of alternation periods of lattice density for solid (s) and porous (p) phases clusters along $\left(L_{\mathrm{s} \|}=L_{\mathrm{p} \|}=110 \mathrm{~nm}\right)$ and perpendicular $\left(L_{\mathrm{s} \perp}=L_{\mathrm{p} \perp}=110 \mathrm{~nm}\right)$ to extension direction (axe s), both.In this case overall porosity of the films equal $50 \%$, so solid and porous phases in the lattice may be considered as co-dimensional.

In the film prepared at $\lambda=44$ regular 3D lattice of lamellar is absent. Along axes, the condition $\xi=L_{\mathrm{s} \|}=L_{\mathrm{p} \|}=$ $110 \mathrm{~nm}$ is valid. However, in transverse direction there is no any coordination between radial functions $g(R)_{s \perp}$ and $g(R)_{\mathrm{p} \perp}$ of a lattice density clusters of phases distribution. In this case the solid and porous phases are non-dimensional ones; the overall porosity is $41 \%$. Non-dimensional character of solid and porous phases is the reason of permeability decreasing of this membrane in 1.5 times in comparison withthe film $(\lambda=78)$ which is characterized by regular 3D lattice of lamellae.

The transition "co-dimensional - non-dimensional" solid and porous phases is related to the change of topological structure of the films, namely, to transition from the model of statistical network of pores to model of oriented network of through flow channels.

\section{2. The influence of the Annealing Temperature on the Structure Transformation at Pore Formation}

The process of the pore formation at uniaxial extension is regulated by self-organization of lamellae and, consequently, strongly depends on the lamellar structure formed at extrusion and annealing stages. Figure 4 shows the relationship of the basic parameters ( $T_{\text {ann }}$ and $\lambda$ ), ensuring the achievement of percolation values of the overall porosity (23 and 30\% for PE and PP films, respectively). This figure shows, under what combination of values $\lambda$ and $T_{\text {ann }}$ appears through permeability in porous polyolefin films.
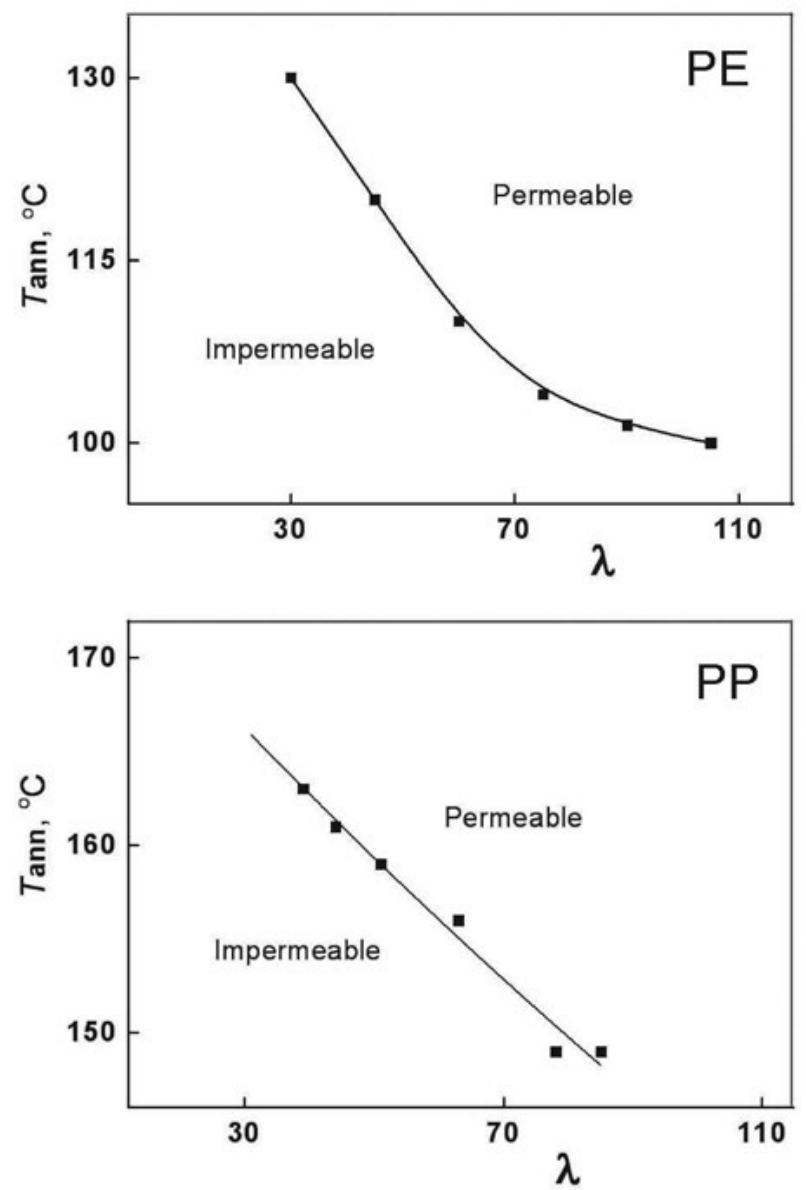

Figure 4. Relation between $\lambda$ and $\mathrm{T}_{\text {ann }}$ at the threshold values of overall porosity for PE and PP films. Uniaxial extension is $200 \%$.

The figure evidences: when extrusion was performed at lower $\lambda$, the percolation value $P^{\star}$ is achieved at higher $T_{\text {ann }}$, and vice versa; if on the extrusion stage greater $\lambda$ was used, then annealing can be performed at lower temperatures $T_{\text {ann }}$. However, this regularity is disrupted for PE. The fact is that structural rearrangement, consisting in increasing the size of lamellae occurs when annealing was carried out at temperatures at which the mobility of the chains in 
the PE crystals appears. According to dynamic mechanical analysis (DMA) for obtained PE films, this temperature is in the range $90-100{ }^{\circ} \mathrm{C}$ (Figure 5). Annealing of the films at lower temperatures does not permit to realize the perfecting of the lamellar structure and transition to a hard-elastic state, which is a prerequisite for the formation the through channels, upon subsequent uniaxial extension. The weakening of the connection between $\lambda$ and $T_{\text {ann }}$ for PE films at $T_{\text {ann }}$ close to $100{ }^{\circ} \mathrm{C}$ shows, that further increase $\lambda$ does not allows to decrease $T_{\text {ann }}$ below this temperature. For PP films character of relation between thresholds $\lambda$ and $T_{\text {ann }}$ does not change, because in this case used values $\lambda$ correspond $T_{\text {ann, }}$, which are higher than appearance of chains mobility in crystals $130-140^{\circ} \mathrm{C}$ according to DMA (Figure 5).

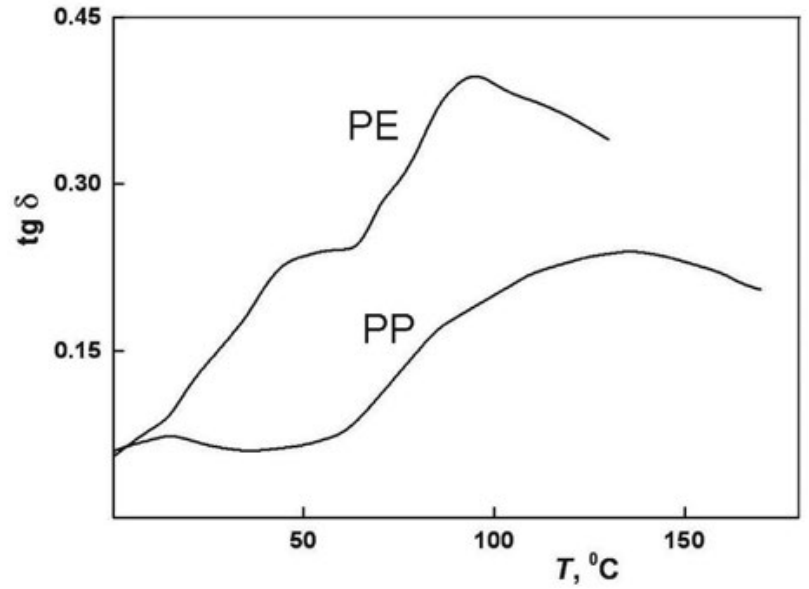

Figure 5. The temperature dependences of tg $\delta$ : PE and PP films.

To establish a correlation between the density of the porous phase $\Omega_{\mathrm{p}}$ (the surface area occupied by all types of pores) and overall porosity $P$, the PP films obtained at different $T_{\text {ann }}$ for which there is a linear relationship between these parameters were selected. In the case of the preva-

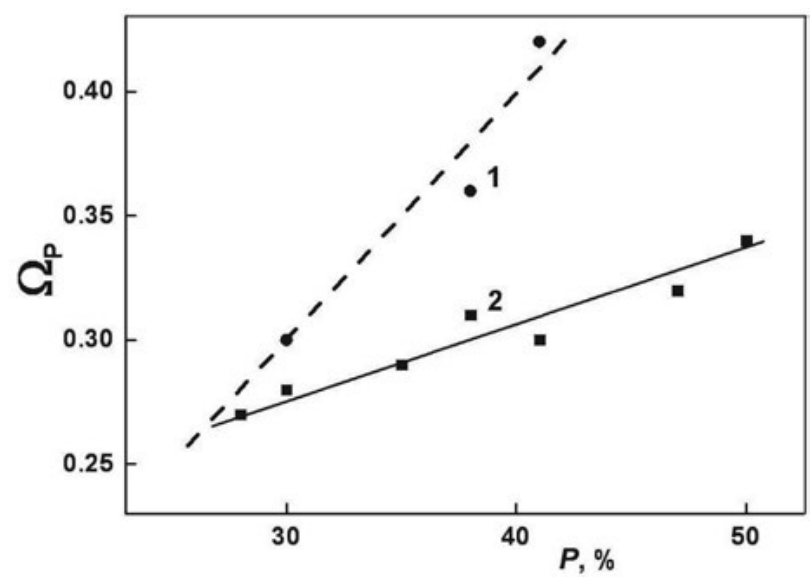

Figure 6. The dependences of the average lattice density $\Omega_{\mathrm{p}}$ of orous phase clusters on the surface of PE (1) and PP (2) films on overall porosity $\mathrm{P}$. The dashed line corresponds to $\Omega_{\mathrm{p}}=\mathrm{P}$. lence of the through oriented channels (after the percolation threshold) occurs a linear correlation between two and three dimensional porosity (Figure 6).

The dependence of permeability of porous films $G$ on the parameter $\tau=\left(P-P^{\star}\right) / P^{\star}$ characterizing the degree of deviation of the porosity parameter $P$ from the percolation threshold $P^{\star}$ was studied. The parameter $P$ in the region $P>P^{\star}$ was varied by changing of $T_{\text {ann }} ; P$ linearly increases with $T_{\text {ann. }}$. It was shown that the dependence $G(\tau)$ is a power-law function $G \sim \tau^{t}$ and is characterized by the critical indices $t=1.5$ and $t=1.9$ for $\lambda=44$ and $\lambda=78$, respectively.

The change of the critical index $t$ with increasing $\lambda$ can be associated with the transition from percolation in a random inhomogeneous medium $(t=1.5)$ to anisotropic percolation over oriented through channels $(t=1.9)$. Such a transition should be a consequence of an increase in the cooperativity of the lamellae ordering process at the stage of pore formation and the development of a regular spatial 3D lattice of particles.

An analysis of the two-dimensional SEM images of the membrane surface makes it possible to clear up the character of pore formation process with increasing porosity $P$ or the degree of deviation $\tau$ from the percolation threshold $P^{*}$. The areas occupied by pores increase that corresponds to the pore growth under steady-state conditions at which the lamellar structure of membranes is ordered.

The investigation of correlations between the two-dimensional network density $\Omega_{\mathrm{p}}$ of porous phase clusters and the volume porosity $P$ of PP membranes shows that $\Omega_{\mathrm{p}}$ is independent of $P$ in the case of $\lambda=44$; at $\lambda=78$ the linear correlation between these parameters is observed. The equality $\Omega_{\mathrm{p}}=P$ is valid for homogeneous systems. Porous phase clusters in the membranes under study are topologically inhomogeneous due to the presence of three pore types: through, closed, and open cell. The establishment of the linear dependence $\Omega_{\mathrm{p}}(P)$ in going from $\lambda=44$ to $\lambda=78$ suggests that, among three pore types, through channels begin to dominate.

With an increase in $T_{\text {ann }}$, lamellae undergo ordering: the larger is the value of $\lambda$, the higher is the rate of decrease in the degree of ordering the lamellae $\varphi$ which approaches zero at $T_{\text {ann }} \rightarrow T_{m}$. The transition from a less ordered cluster of the solid phase to a more ordered cluster is associated with the convergence of the values of the periods $L_{p \|}$ and $L_{s \|}$ and with the formation of a superlattice of lamellae. Similar self-organization of particles in membranes occurs with an increase in the parameter $\lambda$ at a fixed temperature $T_{\text {ann }}$.

The effect of the annealing temperature $T_{\text {ann }}$ on the degree of ordering the lamellae $\varphi$ in the membranes is illustrated in Figure7 (curve 2). In the range $T_{\text {ann }}<439-440$ $\mathrm{K}$ with an increase in $T_{\mathrm{ann}}$, lamellae undergo ordering. In this case, the larger is the value of $\lambda$, the higher is the rate of decrease in the parameter $\varphi$. In the temperature range 
$T_{\text {ann }}>440 \mathrm{~K}$ the parameter $\varphi$ is calculated for aggregates of lamellae. The permeability $G$ of the membranes prepared at the parameter $\lambda=78$ increases to infinity with an increase in the annealing temperature $T_{\text {ann }}$ (Figure 7 , curve 1). This behavior of the dependence $G\left(T_{\text {ann }}\right)$ is explained by the fact that, in the range $T_{\mathrm{ann}}<440 \mathrm{~K}$, the permeability increases as a result of the increase in the degree of ordering of the particle lattice (with a decrease in $\varphi$ ) at a constant pore size $d$, whereas at $T_{\text {ann }}>440 \mathrm{~K}$, it increases due to the increase in the pore size $d$ when $\varphi \approx$ const.

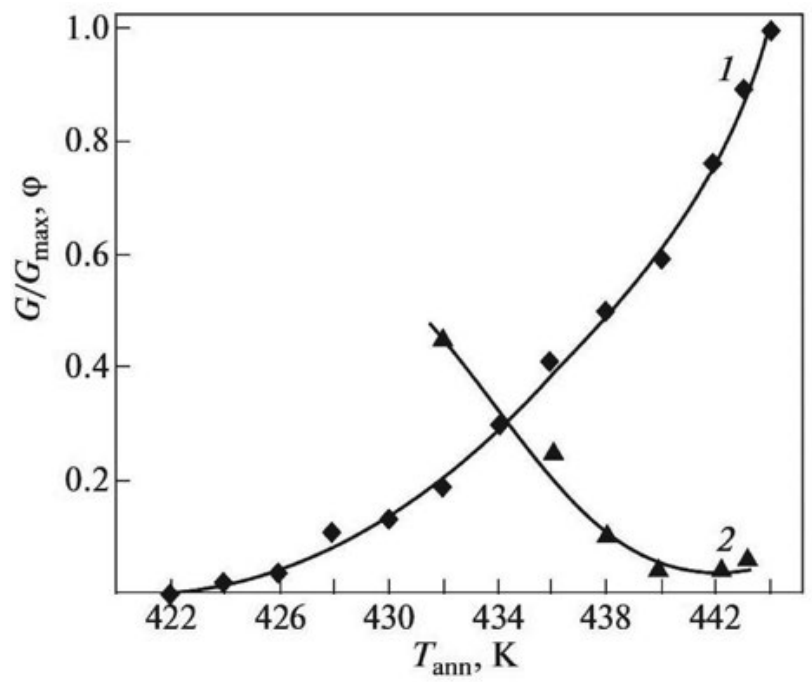

Figure 7. The dependences of the ratio $\mathrm{G} / \mathrm{G}_{\max }$ for the permeability of porous films (1) and the parameter $\varphi$ (2) on the temperature $T_{\text {ann }}$.

The statistical analysis of SEM images of PP porous films prepared at $\lambda=44$ and 78 shows that the films surfaces exhibit polymorphism represented by two regions with different topologies: regularly arranged particles similar to those ones in the films annealed at $T_{\text {ann }}<442 \mathrm{~K}$, and the regions characterized by larger supermolecular structure formed at $T_{\text {ann }}>442 \mathrm{~K}$ whose size increases with $T_{\text {ann }}$. The transformation of the film surface microrelief from the first type to the second one of regions manifests itself as a sharp increase in the period $L_{s \|}$ of alternation of the density of the solid phase cluster (Figure 8).

The increase of $L_{s \|}$ in the films annealed at $T_{\text {ann }}=$ $442-444 \mathrm{~K}$ is associated with the formation of stacks of lamellae constituting the framework of the solid phase percolation cluster. ${ }^{16}$ The aggregation of lamellae leads to the separation of the curves $L_{\mathrm{sl}}\left(T_{\mathrm{ann}}\right)$ at the point of bifurcation at a temperatures $T_{\text {ann }}>440 \mathrm{~K}$ into two branches: for the individual lamellae (curve 3 ) and for the aggregates of lamellae. It should be noted that the alternation of the solid phase density $L_{s||}$ for the individual lamellae, within the error of calculation, does not depend on $T_{\text {ann }}$ and $\lambda$.

Thus, two self-assembly mechanisms with increasing $T_{\text {ann }}$ were detected: the gradual and bifurcation ones; the latter is characterized by a higher cooperativity of the

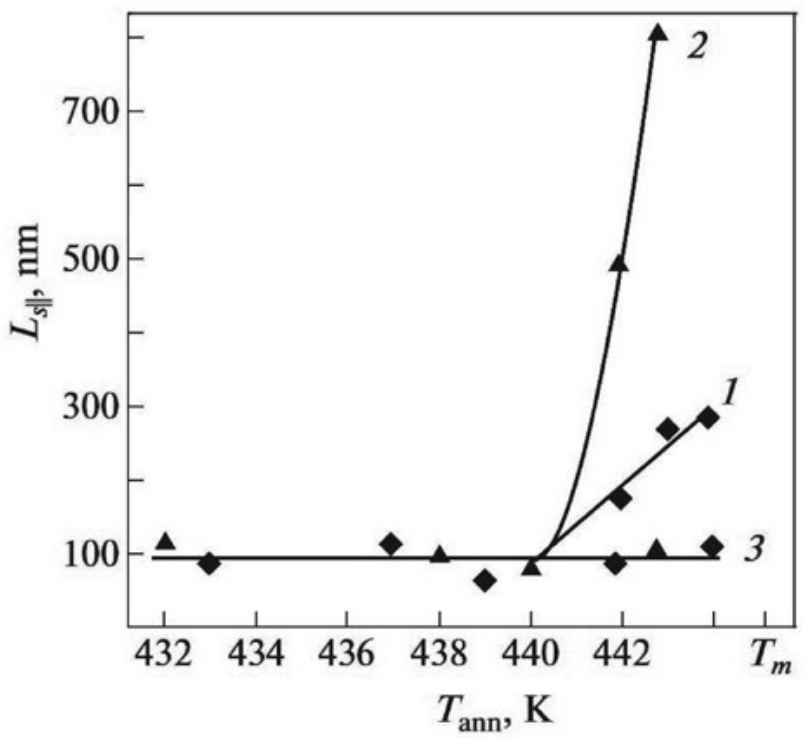

Figure 8. The dependences of period $\mathrm{L}_{s||}$ in PP porous films on the annealing temperature $\mathrm{T}_{\mathrm{ann}}$ for the aggregates of lamellae at $\lambda=44$ (1), and $\lambda=78$ (2), and for the individual lamellae (3).

lamella ordering process. The choice of this or that mechanism by the system depends on the melt spin draw ratio $\lambda$ during extrusion.

\section{3. The Uniaxial Extension Stage}

The self-organization of elements forming the the film surface relief, which is similar to the lamellar structure of the polymer, occurs at the stage of uniaxial extension of the films (pore formation). It should be noted that uniaxial extension of the annealed PE and PP films leads to the formation of pores even at small degrees of deformation $\varepsilon$, immediately from the beginning of the extension process that may be observed visually in the change of transparent annealed films to opaque milk-white microporous material because of the light scattering on the walls of pores. ${ }^{22}$

Uniaxial extension of the polyolefin samples along the direction of extrusion initiates an ordering process of particles of the solid phase - crystalline lamellae - and the ties connecting them. The self-organization of solid phase particles is accompanied by the increase in anisotropy of mechanical properties of the samples, change in their texture from biaxial to axial, an increasing in the degree of homogeneity of the spatial structure and a significant growth in the permeability, which reaches a value of $250 \mathrm{l} /$ $\left(\mathrm{m}^{2} \mathrm{~h} \mathrm{~atm}\right)$ that is characteristic for the membranes with a regular 3D lattice lamellae $e^{2,5,17}$.

\section{Conclusion}

The transformations of supermolecular organization in the process of porous structure formation under study 
have been analyzed using statistical treatment of the SEM images of the PE and PP films surfaces at variations of their orientation degree. The porous structure of these films is originated at uniaxial extension stage following the extrusion and annealling. Basic characteristics of the porous structure - permeability and overall porosity - are significantly affected by both the degree of orientation $\lambda$ of the polymer melt at extrusion stage and also the annealing temperature $T_{\text {ann }}$ of the extruded films. The dependences of permeability on $T_{\mathrm{ann}}$ and $\lambda$ are characterized by the percolation threshold for the appearance of through flow channels. At uniaxial extension the permeability increases with degree of extension due to increasing of number and sizes of through pores. This growth is accompanied by a rising of the ordering degree of the structure elements - self-organization and the formation of the superlattice of crystalline lamellae. The sample structure is transformed from biaxial topology of the random network of pores to uniaxial one of the oriented through channels. The self-organization of porous phase is the result of formation the regular spatial lamellar 3D lattice at the pore formation stage. This transformation may be considered as the disorder-order transition which leads to the ordering of the multilevel supermolecular structure of the film.

\section{Acknowledgments}

This study was supported by Joint Research Projects of the Russian Academy of Sciences and the Faculty for Natural Sciences and Engineering, University of Ljubljana (Slovenia), BI-RU/14-15-037 and BI-RU/16-18-017.

\section{References}

1. J. Kim, S. S. Kim, M. Park, M. Jang, J. Membr. Sci. 2008, 318, 201-209. DOI:10.1016/j.memsci.2008.02.050

2. G. K. Elyashevich, E. Yu. Rozova, E. A. Karpov, RF Patent Number 2,140,936, date of patent April 15, 1997.

3. J. Loboda-Cackovic, H. Cackovic, R. Hosemann, Macromol. Sci. Part B. Phys. 1979, 16, 127-144.

DOI:10.1080/00222347908212286

4. I. K. Park, H. D. Noether, Colloid Polym. Sci. 1975, 253, 824839. DOI:10.1007/BF01452402
5. G. K. Elyashevich, I. S. Kuryndin, V. K. Lavrentyev, A. Yu. Bobrovskii, V. Bukošek, Phys. Solid State 2012, 54, 1907-1916. DOI: $10.1134 /$ S1063783412090090

6. A. V. Yefimov, V. P. Lapshin, V. I. Fartunin, , P. V. Kozlov, N. F. Bakeyev, Polymer Science 1983, 25, 692-700.

7. E. A. Karpov, V. K. Lavrentyev, E. Yu. Rozova, G. K. Elyashevich, Polymer Science 1995, A.37, 1247-1253.

8. R. E. Kesting. Synthetic polymer membranes. A structural perspective. 2nd Edn., John Wiley \& Sons, New York- Chichester, 1985.

9. M. Mulder. Basic Principles of Membrane Technology, Kluwer Acad. Publ., Dordrecht, 1991.

10. S.Y. Lee, S.Y. Park, H.S. Song, Polymer 2006, 47, 3540-3547. DOI:10.1016/j.polymer.2006.03.070

11. S. S. Zhang, J. Power Sources 2007, 295, 351-364. DOI:10.1016/j.jpowsour.2006.10.065

12. I. Novák, G. K. Elyashevich, I. Chodák, A. S. Olifirenko, M. Števiar, M. Špírková, N. N. Saprykina, E. N. Vlasova, A. Kleinová, Eur. Polym. J. 2008, 44, 2702-2707.

DOI:10.1016/j.eurpolymj.2008.05.015

13. A. Bobrovskii, V. Shibaev, G. Elyashevich, E. Rosova, A. Shimkin, V. Shirinyan, A. Bubnov, M. Kaspar, V. Hamplova, M. Glogarova, Liq. Cryst. 2008, 35, 533-539.

DOI:10.1080/02678290802015697

14. G. K. Elyashevich, A. G. Kozlov, E. Yu. Rozova, Polymer Science, Ser. A.1998, 40, 567-573.

15. D. Stauffer, A. Aharony, Introduction to percolation theory, Taylor and Francis, London, 1994.

16. D. V. Novikov, V. K. Lavrentyev, G. K. Elyashevich, V. Bukošek, Phys. Solid State 2012, 54, 1903-1906.

DOI:10.1134/S1063783412090223

17. I. S. Kuryndin, V. K. Lavrentyev, V. Bukošek, G. K. Elyashevich, Polymer Sci. 2015, A57, 717-722.

18. A. Bobrovskii, V. Shibaev, G. Elyashevich, E. Rosova, A. Shimkin, V. Shirinyan, K.-L. Cheng, Polym. Adv. Technol. 2010, 21, 100-112.

19. J. M. Ziman, Models of Disorder, Cambridge University Press, London, 1979.

20. M. Raab, J. Šcudla, A. G. Kozlov, V. K. Lavrentyev, G .K. Elyashevich, J. Appl. Polym. Sci. 2001, 80, 214-222.

21. G. K. Elyashevich, I. S. Kuryndin, E. Yu. Rosova, Polym. Adv. Technol. 2002, 13, 725-736. DOI:10.1002/pat.251

22. A. A. Zinchik, I. S. Kuryndin, K. V. Ezhova, G. K. Elyashevich, Proc. SPIE 2016, 9890, 989016 (1-7). DOI:10.1117/12.2227776.

\section{Povzetek}

Na mikroporoznih poliolefinskih (polietilenskih in polipropilenskih) filmih, pripravljenih v postopku ekstruzije iz taline polimera, čemur je sledilo žarjenje, enoosno raztezanje ter toplotno stabiliziranje, so bili s statistično analizo slik elektronske mikroskopije površja filmov, raziskani strukturni prehodi in mehanizem nastajanja super-rešetk lamel. Strukturo poroznih filmov, pripravljenih v večstopenjskem postopku, smo preučevali z meritvami SEM, gravimetrije in prepustnosti. Pokazano je bilo, da nastajanje por v fazi enoosnega raztezanja spremlja urejanje lamel, njihovo samoorganizacijo nadzira predilno raztezno razmerje ter temperatura žarjenja. Ugotovljeno je bilo, da povečanje teh pokazateljev vodi do prehoda vrste nered - red. Podana je razprava o vplivu pogojev priprave na postopek urejanja pravilne prostorske rešetke lamel. 